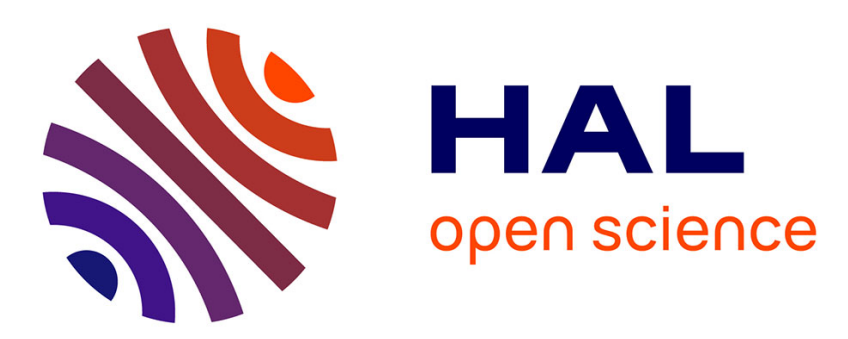

\title{
A reactive decision-making approach to reduce instability in a Master Production Schedule
}

Carlos Herrera, Sana Belmokhtar Berraf, André Thomas, Victor Parada

\section{To cite this version:}

Carlos Herrera, Sana Belmokhtar Berraf, André Thomas, Victor Parada. A reactive decision-making approach to reduce instability in a Master Production Schedule. International Journal of Production Research, 2016, 54 (8), pp.2394-2404. 10.1080/00207543.2015.1078516 . hal-01181276

\section{HAL Id: hal-01181276 https://hal.science/hal-01181276}

Submitted on 5 Aug 2015

HAL is a multi-disciplinary open access archive for the deposit and dissemination of scientific research documents, whether they are published or not. The documents may come from teaching and research institutions in France or abroad, or from public or private research centers.
L'archive ouverte pluridisciplinaire HAL, est destinée au dépôt et à la diffusion de documents scientifiques de niveau recherche, publiés ou non, émanant des établissements d'enseignement et de recherche français ou étrangers, des laboratoires publics ou privés. 


\title{
A reactive decision-making approach to reduce instability in a Master
}

\section{Production Schedule}

\author{
Carlos Herrera \\ Departamento de Ingeniería Industrial, Universidad de Concepción. \\ Avenida Edmundo Larenas 270, Concepción, Chile. \\ Email: cherreral@udec.cl \\ Sana Belmokhtar-Berraf \\ École Supérieure d'Ingénieurs en Électronique et Électrotechnique (ESIEE), France \\ Email: s.berraf@esiee.fr \\ André Thomas \\ Centre de Recherche en Automatique de Nancy, \\ Lorraine University, France \\ Email: andre.thomas@univ-lorraine.fr \\ Víctor Parada* \\ Departamento de Ingeniería Informática, Universidad de Santiago de Chile. \\ Av. Ecuador 3659, Santiago, Chile \\ victor.parada@usach.cl, Tel: (+56)(2) 7180900
}

\begin{abstract}
One of the primary factors that impact the master production scheduling performance is demand fluctuation, which leads to frequently updated decisions, thereby causing instability. Consequently, global cost deteriorates, and productivity decreases. A reactive approach based on parametric mixed-integer programming is proposed that aims to provide a set of plans such that a compromise between production cost and production stability is ensured. Several stability measures and their corresponding mixed-integer programming model are proposed. An experimental study is performed to highlight the effectiveness of the reactive approach with regard to the proposed performance measures. It is observed that an improvement in stability does not mean a significant increase in the total production cost. Furthermore, the procedure yields a set of plans that in practice would enable flexible management of production.
\end{abstract}

Keywords: production planning and control; manufacturing systems; master production schedule; stability; mixed-integer programming. 


\section{Introduction}

A master production program should be periodically adjusted according to demand, and as a consequence, a phenomenon known as nervousness generates instability in the production scheduling. Nervousness is defined as a characteristic in a Master Resource Planning (MRP) system when minor changes in higher level records or the master production schedule cause significant timing or quantity changes in lower level schedules and orders (de Kok and Inderfurth, 1997; Heisig and Fleischmann, 2001). Alternatively, nervousness also can be defined as a shifting of the scheduled setups (Carlson et al., 1979) or as a set of changes in setting up the orders (Blackburn et al., 1986; Ho, 1989). A rolling horizon is used to adjust the master production schedule (MPS) to the demand forecast by minimizing inventory costs with respect to delivery dates. The necessity of reducing nervousness is based on increasing both the throughput times and inventory costs (Heisig, 2002). As a consequence, nervousness causes an increase of the global cost (Steel 1975), a reduction in the productivity (Hayes and Clark 1985) and an increase in the bull-whip effect. Thus, companies have to determine a trade-off considering production costs, quality of service and schedule instability (Blackburn et al. 1986). An important body of work is based on the idea of fixing some decisions by freezing the MPS with the drawback of increasing global cost proportionally to the uncertainty level (Yano and Carlson 1985).

Managing an MRP system requires a decision-making process that considers the MPS level. Reactivity is needed to respond to disturbances by ensuring feasibility of plans and allowing operator involvement in the decision-making process while ensuring the solution quality. When using a rolling planning horizon, between two consecutive schedules, demand changes make previous decisions infeasible; therefore, new decisions have to be made. In practice, decentralized decisions usually based on dispatching rules are most often applied due to their simplicity of implementation. For example, in lean visual management, the performance of operator decisions depends strongly on their limited visibility range which leads to myopic behavior. Thus, recurrent external changes do not allow carrying out predictive planning accurately, and then predictive planning is used as a reference plan in which deviations happen frequently. Although there are several works that study the effectiveness of an MPS, less attention has been devoted to the issue of reactivity 
(Sridharan and LaForge, 1990; Kadipasaoglu and Sridharan 1995; Zhao and Lam 1997). The evaluation of these procedures has often been used with dynamic lot-sizing methods, such as Wagner-within (Blackburn and Millen 1982b) or Silver-Meal algorithms (Blackburn and Millen 1982a). Several studies have tackled the problem with approximate approaches, but only a small number of them are based on exact methods to dampen instability. On the other hand Belmokhtar et al. (2010) proposed the reduction of nervousness in the context of a hierarchical production planning system. To this end, the quantities are disaggregated from the sales and operation plan. Although this approach allows obtaining the required results, the resulting rigid planning contrasts with the flexibility required in real world applications.

Recently the problem of reducing nervousness has focused in the supply chain. For example, Sahin et al. (2008), Robinson et al. (2008) and Nedaei and Mahlooji (2014) propose integration of information between suppliers by rules MPS / AOC. In addition, Pujawan and Smart (2012) and Sahin et al. (2013) conclude that the main causes for nervousness come from the relationship with customers and suppliers. Thus, they proposed tackling the problem from an external perspective instead of using the conventional approach, which is based on simulation and mathematical modeling of internal operations.

In this study, an approach based on a reactive decision-making process to manage nervousness in a production system is studied. This approach generates appropriate production planning without significantly deteriorating the global cost and enabling system operation within an acceptable margin. A set of alternative plans is obtained by minimizing a weighted difference between planned quantities per periods for each item. Because this problem occurs in many industrial companies, the solution proposed to address nervousness would allow such companies to program their productions with less uncertainty and consequently reduce their production costs. The primary interest addresses mass customization companies that should reach a high level of stability with regard to computed schedules for practical convenience.

The study is organized as follows. Related works dealing with instability in planning system and several instability measures are presented in section 2 . The parametric MIP model is presented in section 3, and additional MIP models for each instability measure are also formulated. In section 4 , the effectiveness of the proposed model is shown 
through computational experiments considering different levels of demand variation. Finally, in section 5, the conclusions are presented.

\section{Instability in production planning}

The study of instability in production planning is a critical issue to generate a master production schedule. The works of Steel (1975) and Mather (1977) are among the first dealing with MRP system nervousness. These researchers identify such causes as MPS modifications due to changes in customer orders, lead time, safety stocks and the demand forecast. Carlson et al. (1979) proposed a solution procedure based on a mathematical model to dampen nervousness in a make-to-stock production environment. The authors underline the importance to avoid changes in the first periods of the plan relatively to the more distant ones. In Hayes and Clark (1985), the relationship between instability and productivity was studied. Afterward, the work of Blackburn et al. (1986) examined the effectiveness of alternative strategies in multi-level production processes. Sridharan et al. (1988) studied the problem of how to effectively measure instability and gave one of the more accepted instability measures that is used currently. In the nineties, Inderfurth (1994) studied the nervousness effect in stochastic inventory control. The author defined a measure of nervousness by exclusively taking into account the setup variables considering the setuporiented instability measure as more critical. In Kimms (1998), the author shows the impact of stability using three models of production planning. Furthermore, some approaches are proposed to measure stability by applying them to the MPS solutions. The effects of different cost structures are simulated and analyzed. Finally, an iterative method is proposed to reduce MPS instability.

Several strategies can be implemented to diminish instability in a production system. Three of them have received more attention in the literature: freezing the MPS, end-item safety stock and a lot-for-lot rule for components (Kadipasaoglu and Sridharan, 1995). Frozen intervals consist of fixing quantities within some planning period in which changes are forbidden for the next reschedules. The frozen-period strategy presents the best performance in a stochastic-demand multi-level environment, but in practice such strategies can be used in a complementary way. An extension of this work for a multilevel MRP system instead of a single level with rolling time horizons and a deterministic demand, 
allowed the identification of the impact of lot-sizing rules selection on the MPS freezing parameters (Zhao and Lam, 1997). The same purpose is also considered in Zhao and Xie (1998), where an improved heuristic procedure is compared to the best known lot-sizing rules. In Zhao et al. (2001), freezing the MPS was studied in the case of a multi-item single level with single resource capacity constraints under deterministic demand. Uncertainty in demand is further considered in the context of precedent in Xie et al. (2003), whereas the impact of forecasting error is evaluated in the total cost, schedule instability and system service level. Ho (2008), shows that nervousness in MRP systems strongly depends on both the dampening procedure selected and on the close relationship between operational performance and responsiveness of the planning system.

Tunc et al. (2013) have proposed an approach to assess the nervousness cost by considering a setup-oriented measure by means of three inventory control strategies: static uncertainty, dynamic uncertainty and static-dynamic uncertainty. Interesting findings show that setup-oriented nervousness could be completely eliminated at minor expense, whereas a quantity-oriented measure is difficult to reduce without large cost penalty. The instability of a production system can also be reduced using modified versions of the Wagner-Within and Silver-Meal methods or using models based on mixed integer programming (MIP) that consider previously scheduled periods (Kazan et al., 2000). The results are analyzed in a computational study in which the model based on MIP obtains good stability results in some cases.

Several causes seem to be involved in the origin of instability in a production system. Some of them are: the cost structure, the raw materials costs, the uncertainty level of demand and the rules to define lot-sizing (Kadipasaoglu and Sridharan, 1995). Additionally, it has been suggested that schedules are more stable when there are component commonalities in the supply chain, when the capacity is not tight and when the setup costs are relatively low (Meixell, 2005). The coordination of activities when managing the supply chain has also been identified as a critical factor to instability (van Donselaar et al., 2000). Theoretical studies appropriately reflect what occurs in real world situations in which similar factors affecting instability have been identified, such as lotsizing rules, uncertain demand, item bills and delivery of raw materials (Pujawan, 2004).

Different measures can be used to evaluate the instability numerically (Sridharan et 
al., 1988; Kimms, 1998; Pujawan, 2004; Kabak and Ornek, 2009). A rolling planning horizon $n$, is often used with a specific periodicity $t$ (cycles). Rescheduling is useful for several reasons, such as for planning raw material delivery and adjusting demand to resource capacity. Quantity-oriented instability is given by the differences between scheduled quantities by the MPS in different cycles. Table 1 shows an example of scheduled quantities given by the MPS, where quantity $Q_{i t}^{k}$ represents the scheduled production quantity for an end item $i$, for period $t$, at cycle $k$. In this example, parameters are a rolling horizon, $n=4$, and periodicity in the number of cycles, $\Delta t=1$. For period $t=$ 5, instability is equal to the differences among $\boldsymbol{Q}_{i 5}^{\mathbf{5}}, \boldsymbol{Q}_{i \mathbf{5}}^{\mathbf{4}}, \boldsymbol{Q}_{\boldsymbol{i} \mathbf{5}}^{\mathbf{3}}$ and $\boldsymbol{Q}_{\boldsymbol{i 5}}^{2}$. Note that at cycle 5 the final decision corresponds to $\boldsymbol{Q}_{\boldsymbol{i 5}}^{\mathbf{5}}$ and not to the previous potential decisions computed in cycle 2, 3 and 4. Nevertheless, at cycle 2, planning was done on the basis of potential decision, $\boldsymbol{Q}_{\boldsymbol{i} \mathbf{5}}^{\mathbf{3}}$ which leads to ordering raw materials and planning the corresponding load on the machines. The measure proposed by Sridharan et al. (1988) has been extensively used as a performance criterion in several works, such as those by Zhao and Lam (1997), Xie et al. (2003, 2004). In the following, $N G_{K}$ is introduced as a measure of global instability. It corresponds to a proportional cumulative quantity difference for all periods of the planning horizon, and it is expressed in Eq. (1).

\section{Insert Table 1 about here}

$$
N G_{k}=\max _{i}\left\{\frac{1}{o_{i T}} \sum_{l=1}^{n-1} \sum_{t=k}^{\mathrm{t} \prime}\left|Q_{i t}^{k}-Q_{i t}^{k-l}\right|\right\}_{i=1}^{m}, \forall k \geq n,
$$

where, $t^{\prime}=k+(n-1)-1$ and,

$i$ : end item,

$t$ : time period,

$k$ : rescheduling cycle,

$Q_{i T}^{k}:$ scheduled quantity for end item $i$, period $t$ at cycle $k$,

$n$ : planning horizon length,

$O_{i k}$ : cumulative demand volume of item $i$ from the current cycle $T$ to the rest of the planning horizon $T+n$ such that $O_{i k}=\sum_{t=T}^{T+n} d_{i t}$ where $d_{i t}$ is demand for item 


\section{$i$ at period $t$.}

In contrast to Sridharan et al. (1988), in Eq. (1) there is no weight to reduce the importance of distant quantity differences. Indeed because the earliest decisions are already rescheduled several times, their deviation from the precedent values is further considered. The second reason is that all potential quantities generate several engagements as purchase orders for raw materials. Thus, the proposed measure considers all periods of the planning horizon with the same weight. This measure allows reporting the differences from the previous plans to the demand volume, obtaining a proportional estimation of quantity variation, which is more significant than an absolute variation. The proposed measure is determined by the most unstable product to consider the worst case among the whole group of products.

In addition to the global measure and due to a short rescheduling interval $(\Delta t=1)$, we define a local instability measure to focus on one period only. This measure is provided to help the decision-maker manage the supply process. A precise indication of the level of variation for each purchase order is needed to minimize the difference between purchasing cost and production cost. This local measure $N L$ expressed in Eq. (2) represents quantity differences between the current plan and the previous ones for only the first period in the corresponding cycle. Regarding the precedent global measure, the local measure is proportional to the demand volume to obtain comparable values.

$$
N L_{k}=\max _{\mathrm{i}}\left\{\frac{1}{o_{i k}} \sum_{l=1}^{\mathrm{n}-1}\left|Q_{i k}^{k}-Q_{i k}^{k-l}\right|\right\}_{i=1}^{m}, \forall k \geq n,
$$

Additionally, a smoothing measure is determined by the worst case among the products instability measures. More precisely, it is defined as the differences between production quantities for the same cycle (e.g., in Table 1 for $k=2$, differences between $Q^{2}{ }_{i 2}, Q^{2}{ }_{i 3}, Q^{2}{ }_{i 4}, Q^{2}{ }_{i 5}$ ). So, the smoothing measure can be formulated as follows:

$$
I_{k}=\max _{i}\left\{\sum_{t=k}^{\mathrm{t}^{\prime \prime}}\left|Q_{(t+1)}^{k}-Q_{i t}^{k}\right|\right\}_{i=1}^{m}, \forall k \geq n,
$$

where $t^{\prime \prime}=k+n-1$.

This measure represents an average of the scheduled quantity difference (between 
each period and its immediate next period at each cycle k). In many manufacturing systems these differences tend to be minimized because of the associated costs relating to setup labor turnover, raw material management, etc.

\section{Considered models}

The proposal to measure instability in a production system takes into account four optimization problems: a capacitated lot-sizing problem (CLSP), a smoothing production problem (SPP) and two instability minimization problems. A solution for the CLSP provides a minimum direct-cost production plan. With this plan, it is possible to evaluate the allowed deterioration in the system in terms of the additional cost necessary to produce under a smooth production plan. In particular, the model allows evaluating the additional cost due to smoothing production.

\section{Capacitated lot-sizing problem for the MPS}

The mathematical formulation for this problem specifies a minimum direct-cost production plan that considers the costs of production, inventory, backorder and setup costs. First, the decision variables and input data are defined, and then the model is formulated by equations (4) to (10).

Variables:

$f_{P}^{k} \quad$ objective function for model $M_{P}$ in cycle $k$,

$f_{I}^{k} \quad$ : objective function for model $M_{I}$ in cycle $k$,

$f_{N G}^{k} \quad$ : objective function for model $M_{N G}$ in cycle $k$,

$f_{N L}^{k} \quad$ : objective function for model $M_{N L}$ in cycle $k$,

$x_{i t} \quad$ : production quantity of item $i$ in period $t$,

$s_{i t} \quad$ : inventory of item $i$ in period $t$,

$r_{i t} \quad:$ backlog of item $i$ in period $t$,

$y_{i t} \quad:$ setup for item $i$ in period $t\left(y_{i t}=1 \Leftrightarrow x_{i t}>0, \forall i, \forall t\right)$,

$w_{i t} \quad$ : smoothing production variable of product $i$ in period $t: t>1$.

$z_{i t}^{l} \quad:$ auxiliar variable that represents quantity differences in absolute value Input data:

$d_{i t} \quad$ : demand of item $i$ in period $t$, 
$p_{i t} \quad$ : production cost of item $i$ in period $t$,

$h_{i t} \quad$ : inventory cost of item $i$ in period $\mathrm{t}$,

$b_{i t} \quad$ : backorder cost of item $i$ in period $t$,

$q_{i t} \quad:$ setup cost of item $i$ in period $t$,

$Q_{i t}^{k} \quad$ : production quantity of item $i$ in period $t$ scheduled in cycle $k$,

$C_{t} \quad$ : available capacity of critical resource in period $t$,

$\alpha_{i} \quad$ : marginal consumption of capacity by production of item $i$,

$\beta_{i} \quad$ : marginal consumption of capacity by setup of item $i$,

$M \quad$ : upper bound on production quantity; it is the cumulative demand on the planning horizon.

$$
M_{P}: \quad \min f_{P}^{k}=\sum_{i=1}^{m} \quad \sum_{t=k}^{t^{\prime \prime}}\left(p_{i t} x_{i t}+h_{i t} s_{i t}+b_{i t} r_{i t}+q_{i t} y_{i t}\right)
$$

Subject to:

$$
\begin{array}{r}
s_{i 0}=s_{i n i}, r_{i 0}=r_{i n i}, i \in[1, \ldots, m], \\
s_{i t^{\prime \prime}}=0, r_{i t^{\prime \prime}}=0, i \in[1, \ldots, m], \\
s_{i(t-1)}-r_{i(t-1)}+x_{i t}=d_{i t}+s_{i t}-r_{i t}, i \in[1, \ldots, m], t \in\left[k, \ldots, t^{\prime \prime}\right], \\
x_{i t} \leq M y_{i t}, i \in[1, \ldots, m], t \in\left[k, \ldots, t^{\prime \prime}\right], \\
\sum_{i=0}^{m} \alpha_{i} x_{i t}+\beta_{i} y_{i t} \leq C_{t}, t \in\left[k, \ldots, t^{\prime \prime}\right], \\
x_{i t}, s_{i t}, r_{i t} \geq 0, \quad y_{i t} \in\{0,1\} .
\end{array}
$$

The model $M_{P}$ considers a fixed charge cost assumption, i.e., we suppose a high setup cost that represents the equipment installation and preparation costs (Pochet and Wolsey 2006). Moreover, backorders variables are considered because demand usually exceeds available capacity. The interval of periods $\left[k, \ldots, t^{\prime \prime}\right]$ and the $k$-index in each function, has been explicitly chosen to highlight the rolling horizon nature of the model runs, where, $k$ and $t^{\prime \prime}=k+n-1$ represent, respectively the first and the last period in the rolling horizon. The objective function (4) minimizes production, inventory, backorders and setup cost. Constraint (5) sets the initial inventory and backorders resulting from last production period. Constraint (7) represents the inventory balance, constraint (8) is the relationship between production and setup $\left(y_{i t}=1 \Leftrightarrow x_{i t}>0\right)$, and constraint (9) sets the available capacity by period. 


\section{Smoothing production problem}

A smoothing production problem $\left(M_{I}\right)$ is formulated as a capacitated lot-sizing problem by considering a penalty due to variations in the production quantities between two consecutive periods. The $M_{I}$ problem is described in equations (11)-(14) including the constraints from $M_{P}$. The penalty function is expressed in terms of a set of continuous variables corresponding to such differences (11). The parameter $\lambda$ weighs the differences. Constraints (12) and (13) express the absolute value of any difference in production. Thus, the reformulated objective function $f_{I}$ minimizes both direct production cost $f_{P}$ and cumulative quantity differences between consecutive periods for all items.

$$
M_{I}: \quad \min f_{I}^{k}=f_{P}^{k}+\lambda \sum_{i=1}^{m} \sum_{t=2}^{n} w_{i t}
$$

Subject to:

$$
\begin{array}{r}
(5),(6),(7),(8),(9), \\
x_{i(t+1)}-x_{i t} \leq w_{i t}, \quad i \in[1, \ldots, m], t \in\left[k, \ldots, t^{\prime \prime}\right], \\
x_{i t}-x_{i(t+1)} \leq w_{i t}, \quad i \in[1, \ldots, m], t \in\left[k, \ldots, t^{\prime \prime}\right], \\
x_{i t}, s_{i t}, r_{i t}, w_{i t} \geq 0, \quad y_{i t} \in\{0,1\} .
\end{array}
$$

\section{Instability minimization problems}

Instability minimization problems are formulated to obtain the best possible reduction for the instability measures $N G$ and $N L$. To obtain tight production values the production cost $f_{P}$ is relaxed by allowing a minor deviation, and it is included as a constraint in the way of $\varepsilon$ constraint programming (Ehrgott, 2000). Thus, the larger value is the deviation from production cost; the maximal value is the potential instability gain. In other words, the cost becomes more expensive as instability is reduced. In addition, when the deviation from the initial direct production cost is minimal, the resolution of the problem becomes more difficult due to the tight added constraint that increases time consumption. Then, a trade-off has to be found between the instability reduction needed and the acceptable additional cost. A first $M_{N G}$ problem is formulated to optimize the measure $N G$ and is described by equations (15) - (19) including (5) - (9). The problem is formulated for each cycle $k$ with regard to schedules of precedent cycles. In this problem, 
the objective function (15) minimizes the absolute value of the differences between production quantities $x_{i t}^{k}$ at the current cycle $k$ and those obtained in previous cycles $\left(Q_{i t}^{k-l}\right)$. Constraint (16) allows a cost deterioration in the limit of $\varepsilon \%$. Note that $f_{P}^{k}$, is relative to the computation obtained in the first cycle with the model $M_{P}$, such that the results of the precedent cycle are incorporated as initial conditions into the next cycle. Constraints (17) and (18) guarantee the absolute value for variables $z_{i t}^{l}$.

$$
M_{N G}: \quad \min f_{N G}^{k}=\sum_{i=1}^{m} \sum_{l=1}^{n-1} \sum_{t=k}^{t \prime} z_{i t}^{l}
$$

Subject to

$$
\text { (5), (6), (7), (8), (9) }
$$

$$
\begin{array}{r}
\sum_{i=1}^{m} \quad \sum_{t=1}^{n}\left(p_{i t} x_{i t}^{k}+h_{i t} s_{i t}^{k}+b_{i t} r_{i t}^{k}+q_{i t} y_{i t}^{k}\right) \leq f_{P}^{k *}(1+\varepsilon) \\
x_{i t}^{k}-Q_{i t}^{k-l} \leq z_{i t}^{l}, l \in[1, \ldots, n-1], t \in\left[k, \ldots, t^{\prime}\right], i \in[1, \ldots, m] \\
Q_{i t}^{k-l}-x_{i t}^{k} \leq z_{i t}^{l}, l \in[1, \ldots, n-1], t \in\left[k, \ldots, t^{\prime}\right], i \in[1, \ldots, m] \\
t^{\prime}=n-k-1 \\
x_{i t}^{k}, s_{i t}^{k}, r_{i t}^{k}, z_{i t}^{l} \geq 0, \quad y_{i t}^{k} \in\{0,1\}
\end{array}
$$

In a similar way, a $M_{N L}$ problem specifies the optimal value to measure $N L$, and it is represented by equations (20) - (24) including constraints (5) - (9). The objective function (20) minimizes the differences among quantities of the current period $t$ and those obtained in the previous cycles for the same period (with $\Delta t=1 \Rightarrow t=k$ ). As in the $M_{N G}$, constraint (21) allows the cost deterioration in the limit of a $\varepsilon \%$, and constraints (22) and (23) restrict $z_{i t}^{k}$ to its absolute value.

$$
M_{N L}: \quad \min f_{N L}^{k}=\sum_{i=1}^{m} \quad \sum_{l=1}^{n-1} z_{i t}^{l}
$$

Subject to

$$
\begin{array}{r}
(5),(6),(7),(8),(9) \\
\sum_{i=1}^{m} \quad \sum_{t=1}^{n}\left(p_{i t} x_{i t}^{k}+h_{i t} s_{i t}^{k}+b_{i t} r_{i t}^{k}+q_{i t} y_{i t}^{k}\right) \leq f_{P}^{k *}(1+\varepsilon) \\
x_{i t}^{k}-Q_{i t}^{k-l} \leq z_{i t}^{l}, l \in[1, \ldots, n-1], i \in[1, \ldots, m], t=k \\
Q_{i t}^{k-l}-x_{i t}^{k} \leq z_{i t}^{l}, l \in[1, \ldots, n-1], i \in[1, \ldots, m], t=k \\
x_{i t}^{k}, s_{i t}^{k}, r_{i t}^{k}, z_{i t}^{k} \geq 0, \quad y_{i t} \in\{0,1\}
\end{array}
$$




\section{Experiment description}

The problem instances are generated randomly such that parameter values are close to an industrial case. To obtain an acceptable simulation time, five products are considered. Time periods correspond to weeks; then an operational horizon of $H=52$ periods is chosen to obtain sufficient visibility to measure instability. Note that in practical industrial situations, the operational horizon is determined by the cumulative lead time. The rescheduling interval is $t=1$, and the planning horizon is $n=8$ periods which correspond to two months. The parameter values for production, inventory, backorders and setup are randomly regenerated using a uniform distribution within the following interval: $p_{i t} \sim$ $\mathrm{U}([5,15]) ; h_{i t} \sim \mathrm{U}([10,25]) ; b_{i t} \sim \mathrm{U}([20,40]) ;$ and $q \sim \mathrm{U}([1900,2200])$. Note that parameter intervals have been chosen to enable a possible overlapping between them. Demand has been generated using the normal distribution as $d_{i t}^{k} \sim \eta(\mu, \rho)=\eta(1200, \rho), \forall i, \forall t, \forall k$. To simulate the different levels of variation $(\rho=5,10,15,20(\%))$.

The experiments could be described as three linked processes. In the first process, $M_{I}$ is solved for several values of $\lambda$. Consequently, a set of solutions are obtained, each one corresponding to a different $\lambda$ value. This set of solutions is a set of potential production plans that could be provided to the decision-maker depending on the degree of stability needed and the production cost allowed. The solution with the best trade-off between cost and instability is selected. The proposed approach assumes that this decision is made by the production manager taking into account several factors which typically are, in practice, such as the system capacity, scheduled maintenance, availability of labor, among others. However, the simulation only considers the best trade-off. When alternatives do not arise, we use the original planning, i.e., the standard lot-sizing model (MP).

The second process consists of computation of the set of potential production plans $H$ times, i.e., for all the considered cycles. More precisely, for each cycle, a different instance is generated by varying demand and costs. This data variation simulates the realcase fluctuations that could occur. In the third process, both previous steps are performed to consider several percentage deviations $(\rho)$ from the initial demand. Figure 1 shows a schema of the simulation procedure. For each cycle $k$, all models $M_{P}, M_{I}, M_{N G}$ and $M_{N L}$, are launched independently such that results of the previous cycle are introduced as initial 
conditions of the next cycle (stock and backorders). The strongest variation arises from demand whereas costs remain constant for the rest of the planning horizon. Note that the number of cycle simulations is 60 , and only the last 52 cycles are considered. This allows comparing all periods with the same quantity of information.

\section{Insert Figure 1 about here}

Experiments have been performed for $\rho=\{0.05,0.1,0.15,0.2\}$ supposing that the demand changes following a normal distribution. The generation of instances has been developed using the Python 2.6 programming language, and the MIP models have been solved using GLPK LP/MIP Solver v4.45.

\section{Computational results}

The additional cost due to the consideration of instability is not high compared with the improvement in instability. This effect can be observed in Figure 2, which shows the results obtained for two representative instances. The figure shows the variation in the total cost $\left(f_{I}^{*}\right)$, the instability reduction $(I)$ and instability reduction measures $N G$ and $N L$ depending on the variation in $\lambda$. Note that the Y-axis is graduated as a value in the interval $[0.0,1,0]$ such that each measure is normalized with respect to its initial value (the value obtained when $\lambda=0$ ). For example, in Figure 2(a) when $\lambda=5$ the cost increase showed with the curve $f_{I}^{*}$ means that $f_{I}^{*} / f_{P}^{*}$ slightly exceeds 1.0 where $f_{I}^{*}=f_{P}^{*}$ when $\lambda=0$. The profit obtained for instability measure $I$ decreases to $80 \%$ relatively to its initial value, as seen in the $I$ curve in Figure 2(a) when $\lambda=5$ compared to $\lambda=0$, which is the production difference given by $M_{P}$.

\section{Insert Figure 2 about here}

The proposed approach yields a set of plans that allow managing production flexibly. Each solution corresponds to a fixed value of parameter $\lambda$; therefore, all of these solutions present a trade-off between production cost and instability (Figure 2). Next, the planning can be adjusted flexibly bearing in mind other conditions that typically arise in real situations and that are not explicitly expressed in the mathematical models. In general, 
stability is not desired when it highly deteriorates the production cost $f_{P}^{*}$ which leads to restriction of the $\lambda$ value to an interval delimited by an upper bound and a lower bound. In this way, $\lambda_{\max }$ can be used to identify the interval upper bound corresponding to the acceptable cost deterioration. Analogously, $\lambda_{\min }$ can be used to define the minimal instability reduction needed corresponding to the minimal stability threshold. This range must be defined by taking into account the computational time. The idea is to produce a sufficient amount of production plans in a reasonable amount of computer time. In practice, the interval size is determined in an experimental way such that the runs are stopped as the production cost limit is reached.

A comparison of model performances regarding the production smoothing $(I)$, instability $(N G, N L)$ and production cost increase is presented. Because instability addresses cycle rescheduling, the experimentation phase is then performed for several cycles of the operational horizon thus, a focused analysis is proposed to show the behavior of the different indicators for instances with $5 \%$ demand variation during all cycles.

In spite of a slight cost increase in $M_{I}$, a significant reduction in terms of instability is observed. Figure 3 depicts the results of $M_{I}$ and $M_{P}$ for a demand variation of $5 \%$. It is observed that improvement is achieved using $M_{I}$ compared to $M_{P}$ with regard to instability measures for all periods in the operational horizon of simulation. The experimental results show the positive effect on instability reduction as a consequence of instability minimization obtained with model $M_{I}$. Similarly, though less notable, $N L$ and $N G$ reflect the efficiency of $M_{I}$ model.

\section{Insert Figure 3 about here}

The level of variation in demand is an important factor in determining the production planning. In fact, more variation might involve increased instability and significant changes in the total cost during the planning period. Thus, it is interesting to study whether the behavior detected for a level of demand variation of $5 \%$ holds for other levels. In table 2 , the production cost obtained with the conventional model $M_{P}$ is compared with the proposed models $M_{I}, M_{N G}$ and $M_{N L}$ for $5 \%, 10 \%, 15 \%$ and $20 \%$ of demand variation. The columns in the Table contain the relative deviations from $M_{P}$ values. 
A significant improvement achieved by model $M_{I}$ with regard to instability reduction is observed (Table 2). In fact, model $M_{I}$ achieves up to $93 \%$ of stability, which is obtained with a $5 \%$ of demand variation. Thus, the level of reduction obtained with $M_{I}$ depends on the demand variation. In particular, the higher demand variation is the lesser the instability reduction is, as can be observed in Table 2 experimenting a decrease from $78.52 \%$ to $35.82 \%$. This decrease could be explained by the possibility to smooth production when demand does not greatly exceed the available capacity whereas it becomes much more difficult when demand reaches a peak of variation. Related to model $M_{N G}$, the stability is difficult to achieve because the best reduction does not exceed $30.00 \%$ on average. Nevertheless, when demand variation increases, the difference between models $M_{I}$ and $M_{N G}$ decreases from $64.24 \%$ (78.52-14.28) to $6.39 \%$ (35.82-29.43). The model $M_{N L}$ presents the worst results because it deteriorates even the performance of the conventional CLSP formulation (see the negative deviation computed on the basis of the column $M_{P}$ ).

The numerical results show the superiority of the $M_{N G}$ model because it presents the minimal values with regard to the global instability measure $N G$ for all the experimented instances. Considering the local instability measure, there is no exclusive dominant model because $M_{N L}$ outperforms both $M_{I}$ and $M_{N G}$ on average for the instance with $15 \%$ and $20 \%$ of demand variation. Furthermore, very close results are obtained for the other demand variations that in general, are closer to real-world situations.

\section{Insert Table 2 about here}

\section{Conclusions}

Several MIP formulations have been proposed to increase reactivity in the decision making at the level of master scheduling in production planning. The first model minimizes the instability in addition to production cost to reduce the instability under a rolling horizon. Instability and both global and local instability measures are defined. Then, two MIP models are proposed to reduce global and local instability measures, respectively. A study with an experimental design has been performed to compare the performance of the proposed models regarding smoothness and instability reduction whereas several demand variation levels have been considered. The experimental design aims to highlight the 
flexibility brought in the decision-making process by generating a set of production plans corresponding to several values of the smoothness weighting. Thus, several alternative plans become possible, which guarantee a trade-off between a minimum instability threshold and maximum cost degradation.

From the experimental analysis, it is concluded that an improvement in stability does not mean a significant increase in the total production cost. Furthermore, the procedure yields a set of plans that in practice would allow flexible managing of production. Specifically, a comparison of model performances regarding the production smoothing $(I)$, instability $(N G, N L)$ and production cost increase, shows that in spite of a slight cost increase in $M_{I}$ a significant reduction in terms of instability is observed. In addition, $M_{N G}$ produces plans closer to those generated by $M_{I}$, regarding the plans produced by $M_{P}$. Additionally, the numerical results show the superiority of $M_{N G}$ because it presents the minimal values regarding the global instability measure $N G$ for all tested instances. In this way, the instability minimization appears to be an excellent choice to dampen instability and to maintain a trade-off between the additional cost and smoothness/instability reduction.

This work opens new perspectives for future studies, in which decentralized decisions can be made based on flexibility on the shop floor, especially when disturbances occur. In fact, the operators would participate in facing short-term disturbances, such as machine breakdown or urgent demand. Such systems could be implemented with a multiagent approach in which a neighborhood search could be used to reach close solutions to those obtained in this proposed centralized approach. 


\section{Acknowledgements}

The authors would like to thank the Complex Engineering Systems Institute, ICM:

P-05-004-F, CONICYT: FBO16, DICYT: 61219-USACH, ECOS/CONICYT: C13E04, STICAMSUD: 13STIC-05. 


\section{References}

Belmokhtar, S., Herrera, C. and Thomas, A. "A general approach for hierarchical production planning considering stability", 3rd International Conference on Information Systems, Logistics and Supply Chain (ILS 2010), Casablanca, Morocco, 2010.

Blackburn, J., Kropp, D., and Millen, R., 1986. A comparison of strategies to dampen nervousness in MRP systems. Management Science, 32(4), 413-429.

Blackburn, J. and Millen, R., 1982a. Improved heuristics for multi-echelon requirements planning systems. Management Science, 32(4), 413-429.

Blackburn, J. and Millen, R., 1982b. The impact of a rolling schedule in a multi-level MRP system. Journal of Operations Management, 2(2), 125-135.

Carlson, R., Jucker, J., and Kroop, D., 1979. Less nervous MRP systems: A dynamic economic lot-sizing approach. Management Science, 25(8), 754-761.

de Kok, T. and Inderfurth, K., 1997. Nervousness in inventory management: comparison of basic control rules. European Journal of Operations Research, 1(16), 55-82.

Ehrgott, M., 2000. Multicriteria Optimization. Mongraphs on technical aspects Berlin: Springer.

Hayes, R. and Clark, K., 1985. Explaining observed productivity differentials between plants: Implications for operations research. Interfaces, 15(6), 3-14.

Heisig, G., 2002. Planning stability in material requirements planning system. Berlin: Springer-Verlag.

Heisig, G. and Fleischmann, M., 2001. Planning stability in a product recovery system.OR Spektrum, 23, 25-50.

Ho, C., 1989. Evaluating the impact of operating environments on MRP system nervousness. International Journal of Production Research, 27(7), 1115-1135.

Ho C., 2008. Exploring the compatibility of dampening procedures and lot-sizing rules in MRP systems under uncertain operating environments. International Journal of Production Research, 46(18), 5097-5120.

Inderfurth, K., 1994. Nervousness in inventory control: analytical results. OR Spektrum, $16,113-123$. 
Kabak, K. and Ornek, A., 2009. An improved metric for measuring multi-item multi-level schedule instability under rolling schedules. Computers \& Industrial Engineering, 56, 691-707.

Kadipasaoglu, S. and Sridharan, V., 1995. Alternative approaches for reducing schedule instability in multistage manufacturing under demand uncertainty. Journal of Operations Management, 13, 193-211.

Kazan, O., Nagi, R., and Rump, C., 2000. New lot-sizing formulations for less nervous production schedules. Computers \& Operations Research, 27(13), 1325-1345.

Kimms, A., 1998. Stability measures for rolling schedules with applications to capacity expansion planning, master production scheduling, and lot sizing. Omega, 26(3), 355366.

Mather, H., 1977. Reschedule the reschedules you just rescheduled-way of life for MRP?. Production and Inventory Management, 18(1), 60-79.

Meixell, M., 2005. The impact of setup costs, commonality, and capacity on schedule stability: An exploratory study. International Journal of Production Economics, 95(1), 95-107.

Nedaei, H. and Mahlooji, H., 2014. Joint multi-objective master production scheduling and rolling horizon policy analysis in make-to-order supply chains. International Journal of Production Research, 52(9), 2767-2787.

Pochet, Y. and Wolsey, L., 2006. Production planning by mixed integer programming. New York: Springer New York.

Pujawan, I., 2004. Schedule nervousness in a manufacturing system: a case study. Production Planning \& Control, 15(5), 515-524.

Pujawan, I. and Smart, A., 2012. Factors affecting schedule instability in manufacturing companies. International Journal of Production Research, 50(8), 2252-2266.

Robinson, E., Sahin, F. and Gao, L., 2008. Master production schedule time interval strategies in make-to-order supply chains. International Journal of Production Research, 46(7), 1933-1954.

Sahin, F., Robinson, E. and Gao, L., 2008. Master production scheduling policy and rolling schedules in a two-stage make-to-order supply chain. International Journal of Production Economics, 115(2), 528-541. 
Sahin, F., Narayanan, A. and Robinson, E., 2013. Rolling horizon planning in supply chains: review, implications and directions for future research. International Journal of Production Research, 51(18), 5413-5436.

Sridharan, S., Berry, W., Udayabhanu, V., 1988. Measuring master production schedule stability under rolling planning horizons. Decision Sciences, 19(1), 147-166.

Sridharan, S. and LaForge, R., 1990. An analysis of alternative policies to achieve schedule stability. Journal of Manufacturing and Operations Management, 3, 53-73.

Steel, D., 1975. The nervous MRP system: how to do battle. Production and Inventory Management, 16(4), 83-89.

Tunc, H., Kilic, O.A., Tarim, S.A., Ekşioğlu, B, 2013. A simple approach for assessing the cost of system nervousness. International Journal of Production Economics, 141(2), 619-625.

van Donselaar, K., van den Nieuwenhof, J., and Visschers, J., 2000. The impact of material coordination concepts on planning stability in supply chains. International Journal of Production Economics, 68(1), 169-176.

Xie, J., Lee, T., and Zhao, X., 2004. Impact of forecasting error on the performance of capacitated multi-item production systems. Computers \& Industrial Engineering, 46(2), 205-219.

Xie, J., Zhao, X., and Lee, T., 2003. Freezing the master production schedule under single resource constraint and demand uncertainty. International Journal of Production Economics, 83(1), 65-84.

Yano, C. and Carlson, R., 1985. An analysis of scheduling policies in multiechelon production systems. IEEE Transactions, 17, 370-377.

Zhao, X. and Lam, K., 1997. Lot-sizing rules and freezing the master production schedule in material requirements planning systems. International Journal of Production Economics, 53(3), 281-305.

Zhao, X., Xie, J., Jiang, Q., 2001, Lot-sizing rule and freezing the master production schedule under capacity constraint and deterministic demand. Production and Operations Management, Vol. 10(1), 45-67.

Zhao, X., Xie, J., 1998. Multilevel lot-sizing heuristics and freezing the master production schedule in material requirements planning systems. Production Planning \& Control. 
9(4). 
Table 1. Example of MPS execution in a rolling horizon for end item I

\begin{tabular}{ccccccccc}
\hline $\mathrm{k} / \mathrm{t}$ & 1 & 2 & 3 & 4 & 5 & 6 & 7 & 8 \\
\hline 1 & $Q_{i 1}^{1}$ & $Q_{i 2}^{1}$ & $Q_{i 3}^{1}$ & $Q_{i 4}^{1}$ & & & & \\
2 & & $\boldsymbol{Q}_{i 2}^{2}$ & $\boldsymbol{Q}_{i 3}^{2}$ & $\boldsymbol{Q}_{i 4}^{2}$ & $\boldsymbol{Q}_{i 5}^{2}$ & & & \\
3 & & & $Q_{i 3}^{3}$ & $Q_{i 4}^{3}$ & $\boldsymbol{Q}_{i 5}^{3}$ & $Q_{i 6}^{3}$ & & \\
4 & & & & $Q_{i 4}^{4}$ & $\boldsymbol{Q}_{i 5}^{4}$ & $Q_{i 6}^{4}$ & $Q_{i 7}^{4}$ & \\
5 & & & & & $\boldsymbol{Q}_{i 5}^{5}$ & $Q_{i 6}^{5}$ & $Q_{i 7}^{5}$ & $Q_{i 8}^{5}$ \\
\hline
\end{tabular}


Table 2. Percentage differences among $M_{I}, M_{N G}$ and $M_{N L}$ with respect to $M_{P}$ for $5 \%$ and $10 \%, 15 \%$ and $20 \%$ of demand variation.

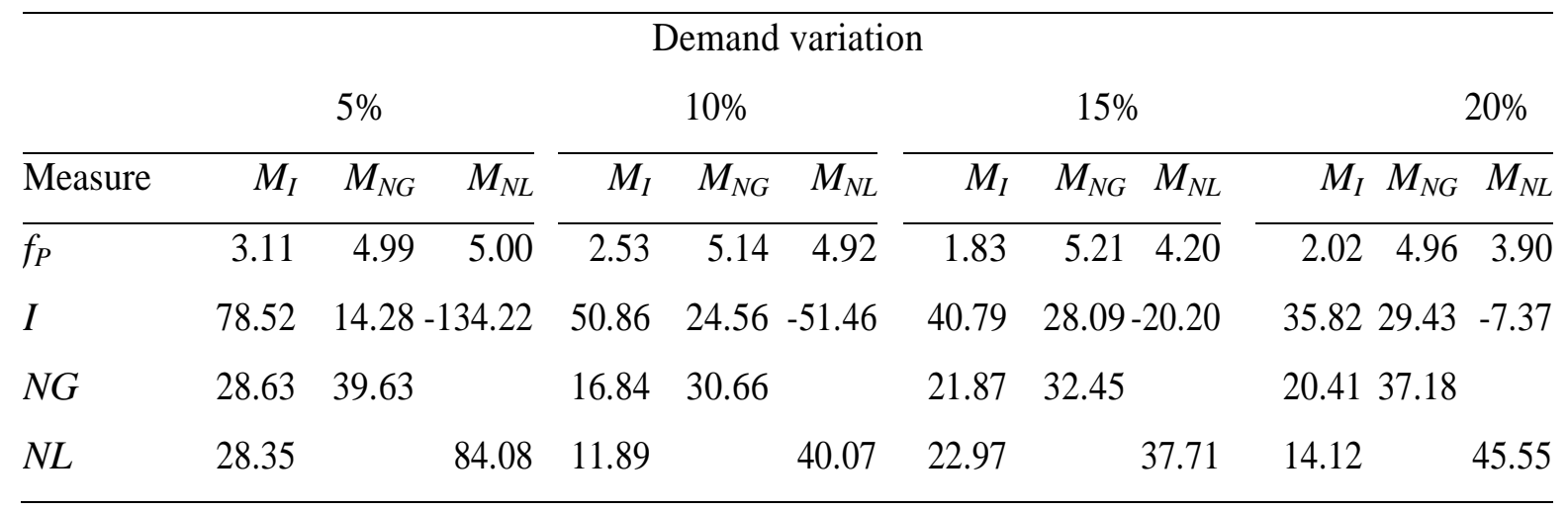




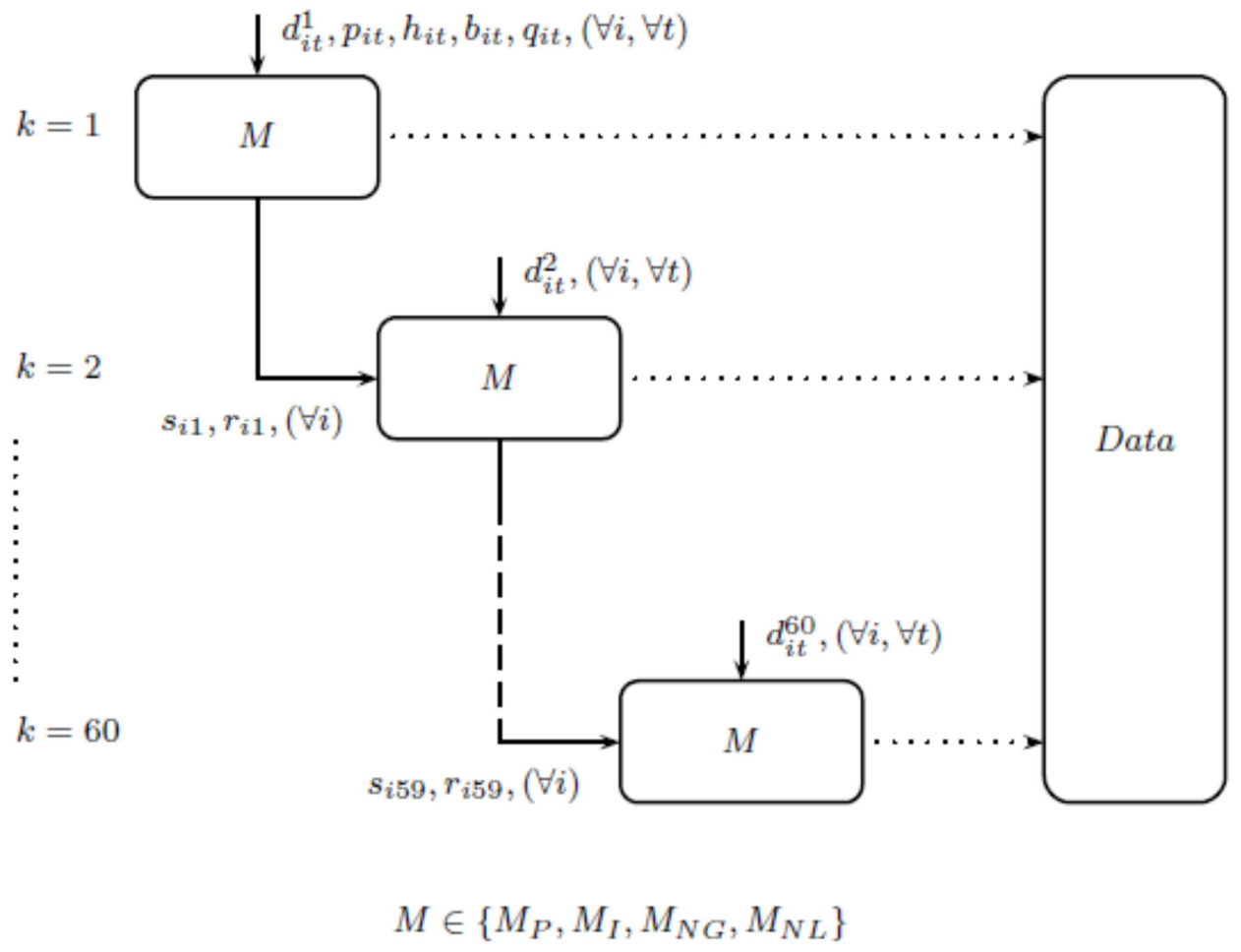

Figure 1. Simulation diagram. 

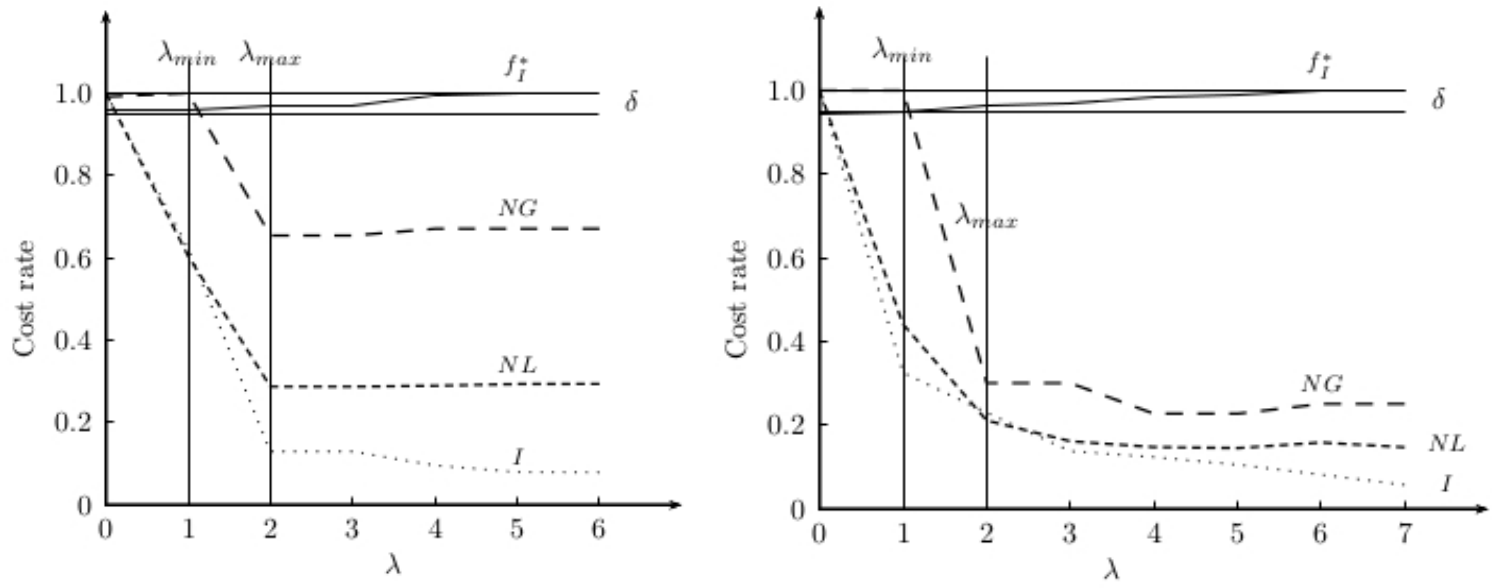

Figure 2. Two example cases of the decision making process: trade-off between total cost $\left(\mathrm{f}_{\mathrm{I}}^{*}\right)$, instability (I) and instability measures (NG and NL). 

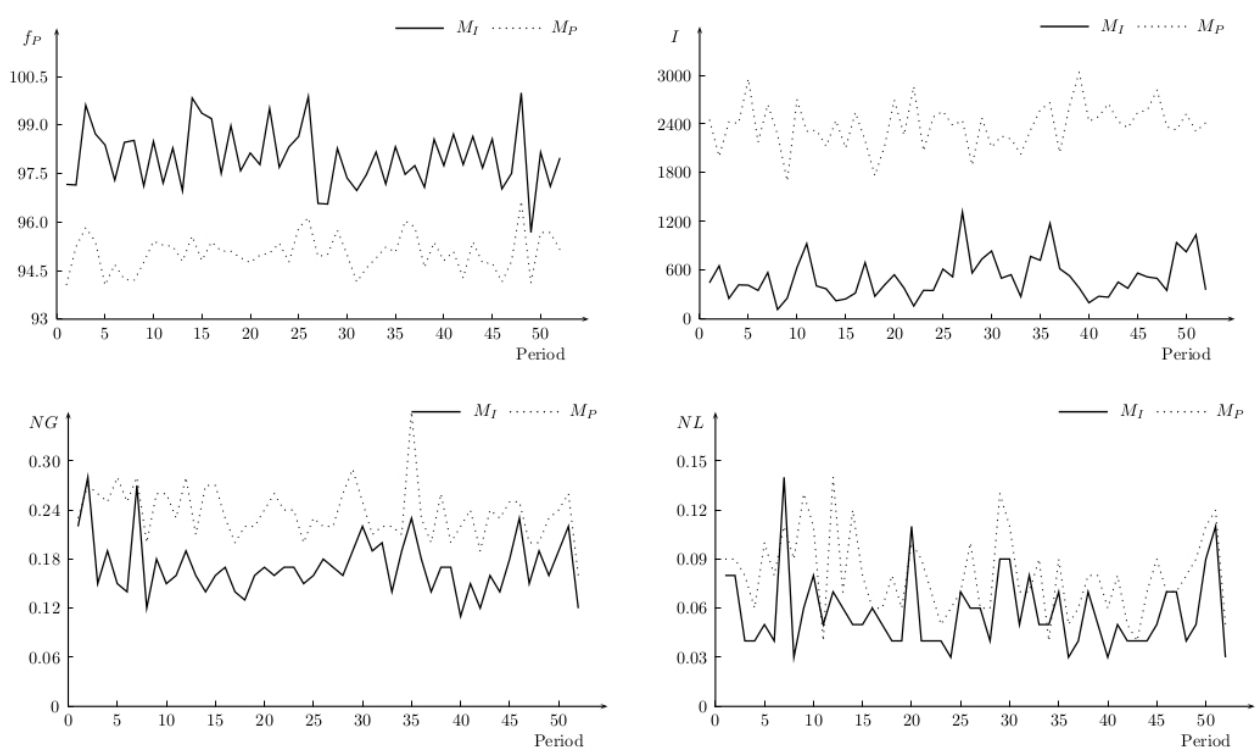

Figure 3. Comparison between $M_{I}$ and considering total cost and instability. 